\title{
What and how to read: Staying current as a young cardiothoracic surgeon
}

\author{
Justin D. Blasberg, MD, ${ }^{a}$ and Seth B. Krantz, $\mathrm{MD}^{\mathrm{b}, \mathrm{c}}$
}

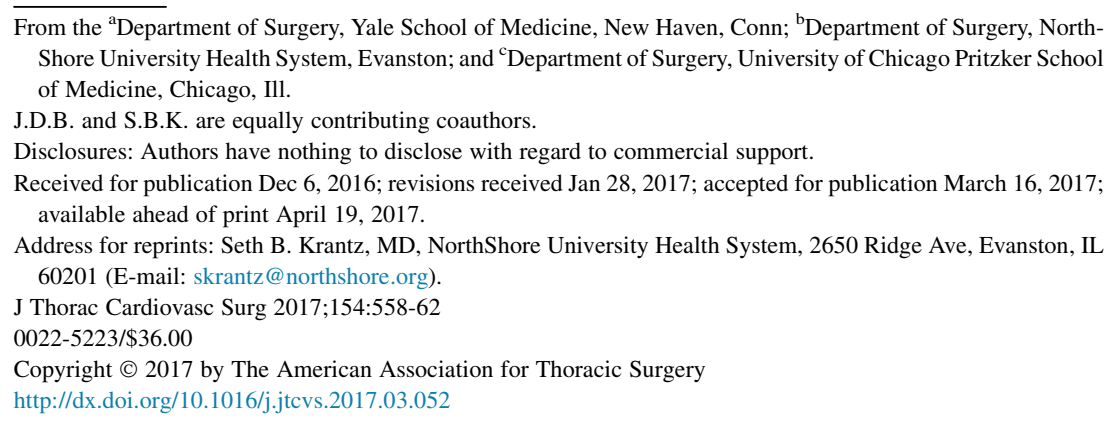

\section{VALUE OF LIFELONG LEARNING}

Lifelong learning is an essential part of the career of any physician, regardless of practice setting, specialty, or academic expectations. The need comes most explicitly from external forces, such as maintenance of certification, hospital credentialing, and academic appointment or advancement, often in the form of formal continuing medical education (CME). ${ }^{1-3}$ Equally important, though, are the internal forces that motivate a the young surgeon to continually improve and expand the knowledge base and to practice in line with the best available evidence. This discipline is emphasized starting on the first day of training and is implicit in the pursuit of becoming an expert in the field of cardiothoracic surgery. These lofty goals and ideals frequently conflict, however, with the stark realities of being a young attending surgeon. Fresh out of training, there is an immediate desire to operate with the frequency and enthusiasm that defines fellowship. During the transition to faculty practice, the desire to read can easily fall by the wayside as the pressures of building a referral base, establishing a rapport with new faculty, and working around block time in the operating room become the new reality. As a young attending surgeon, one is also expected to "pay back" the time with family and friends that was lost during training. During this important transition, it is easy to forget about the need for constant learning. That would be a mistake.

During cardiothoracic surgical training, one learns the basics of all three required disciplines, including disease pathophysiology, anatomy, the "standard" care pathways, and techniques to perform complex operations. Reading as an attending surgeon is drastically different than reading as a trainee, because the objectives are newly focused on clinical scenarios within one's specialty. To obtain a fund of knowledge that is necessary to pass written and oral

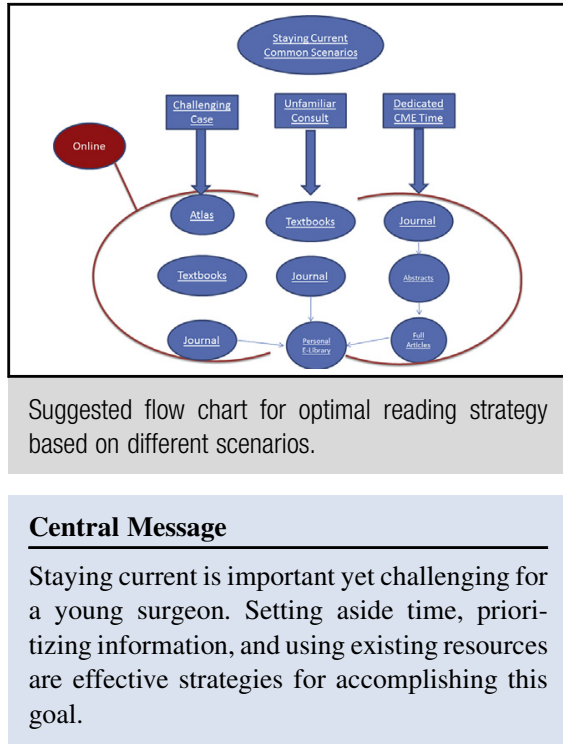

See Editorial Commentary page 563.

board examinations, there is increasingly greater pressure for training programs to have a well thought out and structured educational curriculum, because of the time constraints that limit resident work hours and case requirements, which are more rigorous each year. In the current era, new technology, evolving advancements in surgical outcomes and health services research, and improvements in clinical practice and innovation for both cardiac and thoracic surgical patients have dramatically expanded the foundation of knowledge expected during training. This article seeks to help the young cardiothoracic surgeon, recently out of training, identify what to read, when to read it, and where to look for answers to those most difficult clinical questions the cardiothoracic surgeon will certainly encounter in his or her career (Figure 1).

\section{STAY UP-TO-DATE}

The field of cardiothoracic surgery is constantly evolving, building on the foundation of decades of clinical experience and many "firsts" in the field that have improved the outcomes of our patient population. It is imperative for each surgeon to constantly evolve as well. To stay truly informed, a modern thoracic surgeon must not only stay current in surgical techniques and outcomes but also understand advances in cardiology, oncology, and 


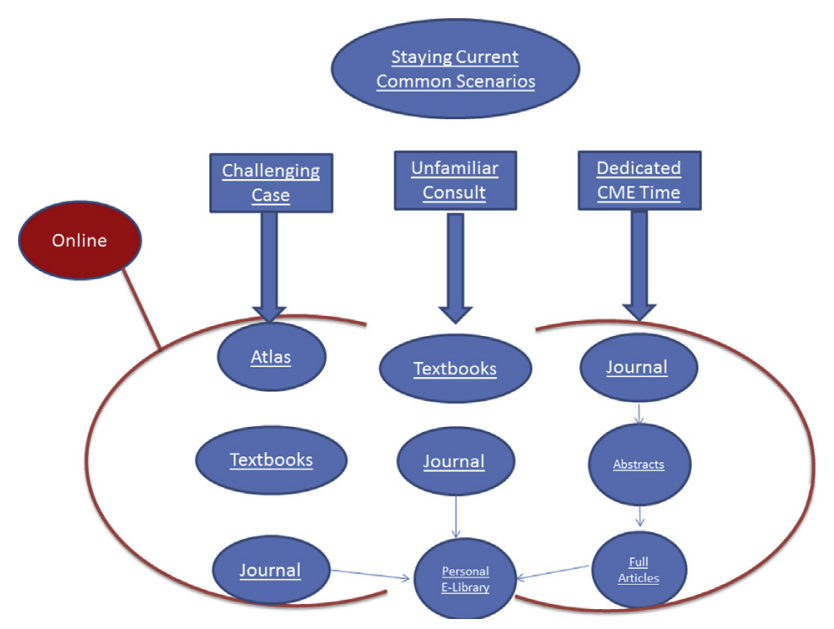

FIGURE 1. Suggested flow chart for optimal reading strategy based on different scenarios. $C M E$, Continuing medical education.

pulmonary critical care. Appropriate reading will therefore cover a wide breadth of journals and other resources, many of which are outlined in Table 1. Understanding what to read and how to expand on the foundation of knowledge one has already obtained requires an examination of how to read. Although a mastery of the existing knowledge is important, identifying how to reference material or where to find the answers to clinical questions may be an equally important goal. As the trainee transitions to faculty practice, developing proper reading habits becomes as critical an attribute as the clinical and technical skills required by this profession.

\section{ADULT EDUCATION AND LEARNING}

The field of adult learning and education continues to provide significant insight into how effectively to accumulate new knowledge. Insights pertinent to a young thoracic surgeon are specifically related to how we absorb high-intensity fact-based materials that we read, how to recall that knowledge in an efficient way, and the best way to be an active reader. Evidence shows that simply reading and rereading is a relatively inefficient use of time. ${ }^{4}$ A more efficient method is active summarization and self-questioning. ${ }^{4-6}$ When reading, the learner should ask questions about what is being read, summarize its key points, and then mentally review the topic. This exercise is relevant in the laboratory setting, ${ }^{4,5}$ in the operating room, and specifically with surgically related knowledge. In a classic example, medical students in anatomy and physiology classes were asked either to read and reread or to read and then actively test themselves on topics in cardiac electrophysiology, endocrinology, and

TABLE 1. List of text and online resources

\begin{tabular}{|c|c|c|c|c|}
\hline Area & Textbooks & Atlases & Journals & Online resources \\
\hline Specialty-wide & $\begin{array}{l}\text { Sabiston and Spencer Surgery } \\
\text { of the Chest (Sellke) } \\
\text { Johns Hopkins Textbook of } \\
\text { Cardiothoracic Surgery } \\
\text { (Yuh) }\end{array}$ & $\begin{array}{l}\text { Operative Techniques in } \\
\text { Thoracic and } \\
\text { Cardiovascular Surgery: A } \\
\text { Comparative Atlas (http:// } \\
\text { www.optechtcs.com) }\end{array}$ & $\begin{array}{l}\text { The Journal of Cardiovascular } \\
\text { and Thoracic Surgery } \\
\text { The Annals of Thoracic } \\
\text { Surgery } \\
\text { Seminars in Thoracic and } \\
\text { Cardiovascular Surgery } \\
\text { European Journal of Cardio- } \\
\text { thoracic Surgery }\end{array}$ & $\begin{array}{l}\text { CTSnet.org } \\
\text { STS.org } \\
\text { AATS.org } \\
\text { TSDA.org } \\
\text { JCTSE WebBrain (jctse. } \\
\quad \text { mrooms.net) } \\
\text { SESATS }\end{array}$ \\
\hline General thoracic & $\begin{array}{l}\text { Pearson's Thoracic and } \\
\text { Esophageal Surgery } \\
\text { (Patterson) } \\
\text { Shields' General Thoracic } \\
\text { Surgery (Shields) } \\
\text { Difficult Decisions in Thoracic } \\
\text { Surgery (Ferguson) }\end{array}$ & $\begin{array}{l}\text { Atlas of Minimally Invasive } \\
\text { Thoracic Surgery } \\
\text { (McKenna) } \\
\text { Thoracic Surgery Atlas } \\
\text { (Ferguson) }\end{array}$ & $\begin{array}{l}\text { Journal of Thoracic Oncology } \\
\text { Chest } \\
\text { Journal of Clinical Oncology } \\
\text { JAMA Oncology }\end{array}$ & \\
\hline Adult cardiac & $\begin{array}{l}\text { Cardiac Surgery in the Adult } \\
\text { (Cohn) } \\
\text { Cardiac Surgery: Safeguards } \\
\text { and Pitfalls in Operative } \\
\text { Technique (Ardehali) } \\
\text { Master Techniques in Surgery: } \\
\text { Cardiac Surgery (Grover) }\end{array}$ & $\begin{array}{l}\text { Cardiac Surgery: Operative } \\
\text { Technique-Expert Consult } \\
\text { (Doty) } \\
\text { Atlas of Cardiac Surgery } \\
\text { Techniques (Sellke) }\end{array}$ & $\begin{array}{l}\text { Circulation } \\
\text { The Journal of Heart and Lung } \\
\text { Transplantation } \\
\text { JACC: Journal of the } \\
\text { American College of } \\
\text { Cardiology } \\
\text { European Heart Journal }\end{array}$ & \\
\hline Congenital & $\begin{array}{l}\text { Comprehensive Surgical } \\
\text { Management of Congenital } \\
\text { Heart Disease (Jonas) } \\
\text { Pediatric Cardiac Surgery } \\
\text { (Mavroudis) }\end{array}$ & $\begin{array}{l}\text { Atlas of Pediatric Cardiac } \\
\text { Surgery (Mavroudis) } \\
\text { Color Atlas of Congenital } \\
\text { Heart Surgery (Litwin) }\end{array}$ & $\begin{array}{l}\text { World Journal for Pediatric } \\
\text { and Congenital Heart } \\
\text { Surgery }\end{array}$ & \\
\hline
\end{tabular}


respiratory physiology. Students who self-tested demonstrated significantly greater recall. ${ }^{6}$ Continual retrieval of the learned knowledge is the critical aspect. In fact, self-testing, which enhances the active recall of pertinent information, is a key component of long-term recall. ${ }^{7}$ These concepts have been reinforced by functional magnetic resonance imaging studies demonstrating that these study strategies activate areas involved with executive control and comprehension, helping link new knowledge to existing knowledge. ${ }^{8}$

\section{DEDICATE THE TIME}

Knowing how to read and what to read is only valuable if one develops appropriate reading habits. First and foremost, developing a schedule is a must. Work schedules for young attending surgeons are constantly changing, and the demands of being on call can be difficult. Establishing a routine that includes regular reading, even if for a short period each week, is essential. Some amount of selflearning during protected time away from the hospital is important.

\section{WHAT TO READ \\ Textbooks}

The existing knowledge base in general thoracic, adult cardiac, and congenital surgical areas is rich and robust. It is somewhat unfair to expect oneself early on to have seen every disease process, to have performed every operation, and to be able to recall the breath of knowledge that one has been exposed to during training. There is no shame in going back to source texts for more obscure topics, such as diseases one is less familiar with, or just to review foundational knowledge. Although textbooks are typically 5 years behind the current knowledge base by the time they are published, they are still relevant as a resource for important for fundamental concepts, historically relevant operative techniques and anatomic descriptions, pearls and pitfalls, and traditional diagnostic and treatment algorithms for commonly encountered clinical scenarios. In addition, review with a reference foundational text is an excellent way to help solidify the knowledge and experienced obtained during training. There are many to textbooks to choose from, and we recommend committing oneself to a single textbook resource for each of the three disciplines: cardiac, thoracic, and congenital surgery. Find whatever text works best for you and stick with it. Table 1 includes a list, although certainly not exhaustive, of valuable textbooks, atlases, journals, and online resources.

\section{Journals}

Innovation and new knowledge in our field are best obtained from peer-reviewed journal articles, regardless of one's specialty. Both of us subscribe to the two major
American journals in thoracic surgery (The Journal of Thoracic and Cardiovascular Surgery and The Annals of Thoracic Surgery) and receive regular E-mail alerts about both new issues and new articles in press. The $E u$ ropean Journal of Cardio-thoracic Surgery provides an additional and incredibly valuable perspective and should also be high on any reading list. Articles in these journals cover a significant percentage of work within our field, including clinical trials, single-institution studies, basic science, and large database reviews. As one reads a given article, it is essential to consider critically how such information might be relevant to practice, whether it might have a significant impact on patient care, and whether the results are believable. The breadth of new information available on a monthly basis may seem overwhelming, and it is important to have a strategy to incorporate this potential onslaught of information efficiently into a busy clinical and academic practice.

Abstracts are your friend. The abstract exists to give you an overview of the larger article. Do not feel guilty about "just" reading an abstract. When new articles or issues comes out, reviewing the abstracts allow the reader to get a complete picture of all the new information. The reader should ask the following question: What is the larger impact of this research question (is this a randomized trial, a large prospective cohort trial, or a retrospective review), and how does this information affect how I take care of patients? In doing so, the reader will be able to prioritize which information should receive a more detailed and in-depth review, which in turn will lead to easier knowledge retention and relevant use in practice. The learning strategies discussed previously can be applied to abstracts as well as complete articles, helping to expand the reader's knowledge base without spending hours reading through every article.

Don't ignore the full article. Although abstracts can help one sort through large amounts of new research, they can never replace the complete article. There are numerous articles to choose from, and because time is limited, we recommend identifying articles that fit into one of two categories: (1) articles of particular relevance to your specific practice and (2) articles that are important within the field and likely to have a significant impact on the current stage of practice. These articles are often defined by their results, the study design, or the size of the trial. Within the article, read critically, identify the research question, focus on the study population, and read through the often-confusing statistical analysis to make sure that things make sense. Pore over the results and tables, and do not ignore the end of the article. The discussion, the invited commentary, and dictated questions during national presentations place 
the results in their proper context. For a young surgeon, the discussion provides a tremendous amount of valuable background and context, including relevant historical trials, related contemporary research, and a broader understanding of how the article's results fit into the broader existing knowledge base. As young surgeons, much of our knowledge is based on textbooks, and we may not have as rich or as intimate an understanding of existing studies as our mentors do. The discussion section serves as a resource to help enrich the reader's understanding of the topic of interest. Finally, the younger reader should get into the habit of saving copies of important, relevant, or seminal articles.

Life is not a closed-book test. Although fundamental aspects of patient care require calling on experience and knowledge at a moment's notice, most clinical practice revolves around a routine that affords the cardiothoracic surgeon an opportunity to review source material. Many of our mentors have large file cabinets filled with printed articles and bookshelves of old volumes of important journals, the pages earmarked and highlighted. Although that tried and true method may be preferred by these seasoned surgeons, technology may significantly organize the reader's personal library of important articles into a more useable format. There are several Web sites and programs (Table 1) that allow one to import articles that have been read, to organize them, and to perform full text searches to recall relevant information. Maintaining a categorized electronic article library, whether on a cloud-based server, an electronic tablet, or on a USB key drive, will create an invaluable personal collection that benefits the surgeon throughout practice. When reading a new abstract or article, use the strategies discussed previously, then add it to one of your electronic libraries, and it will always be at your disposal. You don't have to remember every survival curve and complication rate. With a searchable online database, one needs only to remember how to search for the topic and then simply look it up and review it.

Aggregators aren't cheating. The amount of new articles published on a weekly and monthly basis is astounding. Alerts from relevant journals are helpful but can still lead to information overload. Edited aggregation services exist to help highlight the most impactful and relevant new research. These aggregated sourced, such as he CTSNet journal and news scan (JANS) and Thoracic Surgery News, or similar newsletters, are edited by leaders in the field of cardiothoracic surgery. The knowledge and expertise of these editors can help identify seminal articles and high-impact innovation. These resources provide the citation, and often link to the original article, which can then be read in depth and cataloged as described previously.
Such resources are easy to read, often include articles from trainees and young attending surgeons, and are available at little to no cost.

\section{Online Resources}

In the current era, there is an ongoing expansion of the traditional residency curriculum to address the significant increase in didactic, clinical, and technical skills required by the modern cardiothoracic trainee. Modern residency education acknowledges these challenges and has increased the focus on Web-based educational tools designed to help develop a foundation of knowledge. Supported by The Society of Thoracic Surgeons, this curriculum has been expanded to include the Prerequisite Curriculum, which includes a primer of factual data before the start of cardiothoracic training, as well as the primary curriculum of the Thoracic Surgery Directors Association (TSDA) online training system WebBrain, which has been widely integrated into training programs across the country. These resources are exhaustive and include online readings both from book chapters, seminal articles, and Power Point presentations from annual meetings. Within this resource, the user can find weekly quizzes, case presentations, and videos that highlight the important technical aspects of multiple operations in each of the three disciplines.

We highly recommend the TSDA online curriculum, which is valuable not only for trainees but also for young surgeons. It allows the surgeon the ability to reference all the previously recommended materials in a single format. The TSDA Education Universe is another important resource, designed for trainees but also appropriate for junior staff members. It includes techniques for performing common cardiac procedures such as coronary artery bypass grafting and the inner workings of the heart-lung machine. Thoracic surgical topics include lung cancer staging and advances in molecular classifications, and there are also various topics in the congenital space.

Additional electronic "must haves" include the Thoracic Surgery Resident Association (TSRA) series of downloadable resources. This is an electronic cardiothoracic surgery primer that is fully interactive and provides fundamental knowledge and a good technical foundation for the new trainee. The TSRA Clinical Scenarios in Cardiothoracic Surgery is a fantastic oral board preparation that is also a strong resource for clinical questions, authored by fellow trainees and edited by experts in the field. These authors have found the clinical scenarios app to be one of the most important resources, in addition to the TSRA primer, both during training and early on in practice. The TRSA Web site and app also provide links to other important resources, including podcasts, online journal clubs, and forums to discuss topics important to residents navigating a similar path. Finally, in preparation for board examinations, 
for maintenance of certification, and as a part of ongoing, continual learning, the Self Education Self Assessment in Thoracic Surgery (SESATS) is also an invaluable resource. We used it extensively when preparing for the written section of the boards, and its self-assessment focus is an essential component of successful adult education, as discussed previously.

\section{CONCLUSIONS}

Finding the time and energy to commit to reading and developing a system that includes comprehensive and integrated resources including textbooks, peer-reviewed journal articles, and online resources are fundamental to both obtaining and maintaining a fund of knowledge in cardiothoracic surgery. Given the rigors of cardiothoracic surgery residency and early practice, establishing a schedule that includes time for reading is an essential component not only of the training experience but of one's entire career. These investments pay dividends not only during the cardiothoracic surgery boards but as one joins the workforce and takes on the ultimate responsibility of taking care of patients. Although there are many online and print resources to choose from, each individual must choose which data sources are best suited to his or her preferences. Regardless of the textbook or online database, staying current with novel research, including basic science, translational work, and outcomes is critical to maintaining an evidence-based practice. Committing even a small amount of weekly effort to maintaining a reading effort will pay significant dividends in the future, particularly when pre- sented with a challenging clinical question. Given the wide breath of information that the cardiothoracic surgeon is responsible for, knowing where to look may be even more important than having the answer memorized. There is no question that a Web-based curriculum with interactive resources, phone-based apps, and online forums that allows students and faculty to communicate is the future of our educational system. By investing in an education training experience that includes both the traditional and modern approaches to accessing knowledge, the cardiothoracic trainee is well suited to enter the workforce ready to adapt to future advances.

\section{References}

1. Moffatt-Bruce SD, Ross P, Williams TE Jr. American Board of Thoracic Surgery examination: fewer graduates, more failures. J Thorac Cardiovasc Surg. 2014; 147:1464-9.

2. Johnson DA, Austin DL, Thompson JN. Role of state medical boards in continuing medical education. J Contin Educ Health Prof. 2005;25:183-9.

3. Davis DA, Baron RB, Grichnik K, Topulos G, Agus ZS, Dorman T. Commentary: $\mathrm{CME}$ and its role in the academic medical center: increasing integration, adding value. Acad Med. 2010;85:12-5.

4. Weinstein Y, McDermott KB, Roediger HL. A comparison of study strategies for passages: rereading, answering questions, and generating questions. J Exp Psychol Appl. 2010;16:308-16.

5. McDaniel MA, Howard DC, Einstein GO. The read-recite-review study strategy: effective and portable. Psychol Sci. 2009;20:516-22.

6. Dobson JL, Linderholm T. Self-testing promotes superior retention of anatomy and physiology information. Adv Health Sci Educ Theory Pract. 2015;20:149-61.

7. Karpicke JD, Roediger HL III. The critical importance of retrieval for learning. Science. 2008;319:966-8

8. Moss J, Schunn CD, Schneider W, McNamara DS, Vanlehn K. The neural correlates of strategic reading comprehension: cognitive control and discourse comprehension. Neuroimage. 2011;58:675-86. 